\title{
RECONSTRUÇÃO DA TEMPERATURA E DA PRECIPITAÇÃO MÉDIA ANUAL COM BASE EM ACUMULAÇÕES DE MACRORRESTOS VEGETAIS DA BACIA DO RIO ITANHAÉM, SÃO PAULO, BRASIL
}

\author{
FRANCISCO SANTIAGO (1) \\ Programa de Pós-Graduação em Geociências, Instituto de Geociências, Universidade \\ Estadual de Campinas, Cx.P. 6152, 13083-855, Campinas, SP, Brasil. \\ santiago1403@gmail.com \\ FRESIA RICARDI-BRANCO (D) \\ Departamento de Geologia e Recursos Naturais, Instituto de Geociências, Universidade \\ Estadual de Campinas, Cx.P. 6152, 13083-855, Campinas, SP, Brasil. \\ fresia@unicamp.br
}

\begin{abstract}
Reconstruction of the mean annual temperature and precipitation with basis on plant macrodebris accumulations of the Itanhaém River Basin, São Paulo, Brazil. Paleoclimate reconstruction, specifically of the mean annual temperature (MAT) and the mean annual precipitation (MAP), from fossil angiosperm leaf assemblages, is usually performed using the main leaves physiognomic characteristics, type of leaf margin and leaf area. In order to know the error of these reconstructions, over the years, studies based on the analysis of leaf accumulations from modern forests have been presented. In the southern coast of the São Paulo state, in the Itanhaém River basin, along its main tributaries, especially in the rivers Branco and Preto, which present a meandering pattern, well-preserved plant macrodebris accumulations have been studied. These plant macrodebris accumulations are considered a record of El Niño, the warm phase of the global climate phenomenon El Niño-Southern Oscillation, since they were deposited in periods when this happened, specifically in 1957 and 2004-2005. Based on the analysis of the leaf material of these accumulations, reconstruction of MAT and MAP was carried out. The reconstructions of the MAT underestimated the real value by $1.6^{\circ} \mathrm{C}$ and overestimated it by $0.1^{\circ} \mathrm{C}$. On the other hand, MAP reconstructions only underestimated the actual value, specifically at 620 and $960 \mathrm{~mm}$. The error of these reconstructions seems to be influenced, inter alia, by the habitat, origin habit of the leaf material, sample collection, and not by the occurrence and intensity of El Niño, since during the deposition period of these accumulations, it had no influence on precipitation and temperature.
\end{abstract}

Keywords: paleoclimate reconstruction, leaves physiognomic characteristics, leaf accumulations, modern forests, El Niño.

RESUMO - A reconstrução do paleoclima, especificamente a temperatura média anual (TMA) e a precipitação média anual (PMA), a partir de associações de folhas fósseis de angiospermas, geralmente é efetuada utilizando as principais características fisionômicas das folhas, o tipo de margem e área foliar. Com a finalidade de conhecer o erro destas reconstruções, ao longo dos anos, têm sido apresentados estudos baseados na análise de acumulações de folhas, produto de florestas modernas. No litoral sul do Estado de São Paulo, na Bacia do Rio Itanhaém, ao longo de seus principais afluentes, especialmente nos rios Branco e Preto, os quais apresentam um padrão meandrante, foram estudadas acumulações de macrorrestos vegetais bem preservadas. Estas acumulações são consideradas um registro de El Niño, a fase quente do fenômeno climático global El Niño-Oscilação Sul, já que se depositaram em períodos em que este aconteceu, especificamente em 1957 e 2004-2005. Com base na análise do material foliar dessas acumulações, se levou a cabo a reconstrução da TMA e a PMA. As reconstruções da TMA subestimaram o valor real em $1,6^{\circ} \mathrm{C}$, e o sobrestimaram em $0,1{ }^{\circ} \mathrm{C}$. Por outro lado, as reconstruções da PMA só subestimaram o valor real, especificamente em 620 e $960 \mathrm{~mm}$. O erro destas reconstruções parecem ser consequência de fatores como habitat e hábito de procedência do material foliar, coleta das amostras, entre outros, e não da ocorrência e intensidade de El Niño, já que durante o período de deposição destas acumulações, não teve nenhuma influência sobre as precipitações e a temperatura.

Palavras-chaves: reconstrução do paleoclima, características fisionômicas das folhas, acumulações de folhas, florestas modernas, El Niño. 


\section{INTRODUÇÃO}

As associações de folhas fósseis de angiospermas são geralmente utilizadas para reconstruir a composição e a estrutura das florestas, bem como o clima e o meio ambiente em que se desenvolveram (Burnham, 1989; Burnham et al., 2005). Para a reconstrução do clima, concretamente a temperatura média anual (TMA) e a precipitação média anual (PMA), são comumente utilizadas as principais características fisionômicas das folhas de angiospermas, tipo de margem e tamanho (Wilf, 1997; Wilf et al., 1998; Royer, 2012; Ellis \& Johnson, 2013). Isto se deve ao fato de que existe uma forte covariância entre as folhas com margens sem dentes e a TMA, e entre o tamanho da folha e a PMA (Royer, 2012). Nos últimos anos, diversos estudos têm sido realizados para conhecer a precisão das reconstruções paleoclimáticas, assim como a influência sobre elas da diversidade, do hábito e do ambiente, tomando como referência as folhas de florestas modernas (Wilf, 1997; Wilf et al., 1998; Burnham, 1989, 1997; Burnham et al., 2001, 2005; Ellis \& Johnson, 2013; Ricardi-Branco et al., 2015). Estes estudos têm sido desenvolvidos sobretudo na região tropical, já que muitas associações fósseis que se encontram ao redor do mundo foram derivadas de florestas tropicais ancestrais com grande riqueza de espécies ou apresentaram condições favoráveis para concentrar grande número de espécies derivadas de diferentes unidades ecológicas (Burnham et al., 2005).

A Bacia do Rio Itanhaém, a qual encontra-se no litoral sul do Estado de São Paulo, Sudeste do Brasil, apresenta acumulações de macrorrestos vegetais muito bem preservadas ao longo de seus principais afluentes, especialmente nas curvas dos meandros dos rios Branco e Preto (Ricardi-Branco et al., 2009, 2011; Branco et al., 2011). Estas acumulações de macrorrestos vegetais estão constituídas por material foliar parautóctone, o qual tem sido limitado à sua área de origem (Ricardi-Branco et al., 2009). Por esta razão, a composição taxonômica destas acumulações de macrorrestos vegetais depende da floresta ripária que acompanha o curso do rio e do transporte que sofrem os restos vegetais antes de sua deposição (Ricardi-Branco et al., 2009). Devido ao tipo de sedimento e à química do ambiente subaquático, os quais estão sob a influência das variações climáticas e das marés, as acumulações de macrorrestos vegetais da Bacia do Rio Itanhaém têm-se preservado ao longo do tempo (Branco et al., 2011). Há que se destacar que estas acumulações de macrorrestos vegetais foram depositadas nos períodos de 1957 e 2004-2005 (Ricardi-Branco et al., 2009, 2020). Devido a que estas acumulações se correlacionam com o episódio intenso de El Niño ocorrido no período de 1957-1959 e com o episódio fraco que aconteceu no período de 2004-2005, podem ser consideradas registros de El Niño, a fase quente do fenômeno climático global El Niño-Oscilação Sul (RicardiBranco et al., 2020).

O El Niño-Oscilação Sul é um fenômeno de grande escala, que ocorre no Oceano Pacífico Equatorial, produto do forte acoplamento atmosfera-oceano, que se manifesta sobre a região (Minuzzi et al., 2006). A variação irregular que ocorre em torno das condições normais nas componentes atmosférica e oceânica revela uma fase quente e uma fase fria, as quais são conhecidas como El Niño e La Niña (Minuzzi et al., 2006; Marengo, 2007). A fase quente, El Niño, é um fenômeno que se caracteriza pelo aquecimento anormal das águas superficiais no Oceano Pacífico Tropical, o qual pode alterar o clima regional e global, afetando assim, os regimes de precipitações nas regiões tropicais e de latitudes médias (Minuzzi et al., 2006; Marengo, 2007; Costa, 2012).

Devido ao fato de que o material foliar presente nas acumulações de macrorrestos vegetais da Bacia do Rio Itanhaém preserva suas principais caraterísticas fisionômicas, tipo de margem e área foliar, este trabalho tem como objetivo reconstruir a partir deste material a TMA e a PMA para o momento de sua deposição.

\section{ÁREA DE ESTUdO}

No litoral sul do Estado de São Paulo, no município Itanhaém, se encontra a Bacia do Rio Itanhaém (Figura 1), a segunda maior da costa do estado, com uma superfície de 930 $\mathrm{km}^{2}$ (Camargo et al., 2002). Esta bacia encontra-se sobre uma planície quaternária, que se estende por aproximadamente 50 $\mathrm{km}$ e que tem largura média entre 15 e $16 \mathrm{~km}$ (Camargo et al., 2002; Amaral et al., 2006). Os principais afluentes desta bacia, são os rios Branco e Preto (Figura 1), os quais fluem através de diferentes ecossistemas (Amaral et al., 2006; Ricardi-Branco et al., 2009).

A Bacia do Rio Itanhaém, apesar de apresentar forte ação antrópica, caracteriza-se por preservar remanescentes florestais da Mata Atlântica (IF, 2007), a saber a: (1) floresta ombrófila densa e a (2) formação arbórea/arbustiva-herbácea (IPT/PMI, 2012). A floresta ombrófila densa, a qual ocorre como vegetação primária e secundária (IPT/PMI, 2012), é dividida segundo sua hierarquia topográfica em: (1.1) floresta ombrófila densa montana, (1.2) floresta ombrófila densa submontana e (1.3) floresta ombrófila densa de terras baixas (Veloso et al., 1991). Em contrapartida, a formação arbórea/ arbustiva-herbácea divide-se segundo o seu ecossistema em: (2.1) manguezal, (2.2) vegetação de várzea e (2.3) restinga (IPT/PMI, 2012). Portanto, na área existem ao menos seis tipos de vegetação, sendo a mais importante a restinga, já que abrange maior área, seguida pela floresta ombrófila densa submontana e pela floresta ombrófila densa montana (IPT/ PMI, 2012).

De acordo com a classificação climática de Köppen, a Bacia do Rio Itanhaém está sob a influência de clima Cfa (temperado quente-úmido com verão quente, Sparovek et al., 2007), o qual se caracteriza por apresentar TMA de $24^{\circ} \mathrm{C}$ e PMA de 2120 mm (IPT/PMI, 2012).

\section{MATERIAL E MÉTODOS}

\section{Material foliar analisado}

Neste estudo, foram analisadas duas amostras de material foliar que se encontram resguardadas no Laboratório de Paleo- 


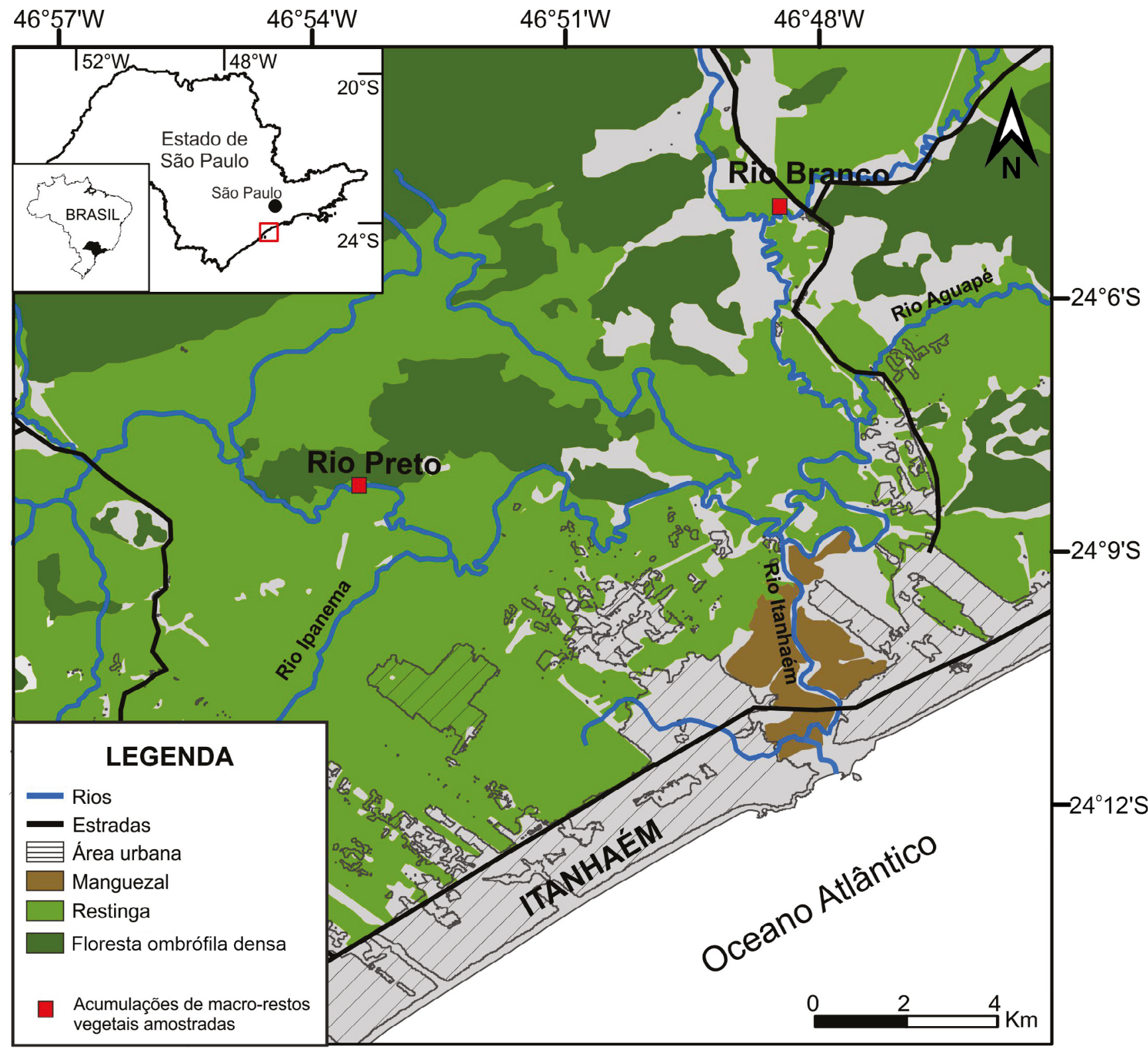

Figura 1. Localização das acumulações de macrorrestos vegetais amostradas na Bacia do Rio Itanhaém (modificado de Ricardi-Branco et al., 2009).

Figure 1. Location of the plant macrodebris accumulations sampled in the Itanhaém river basin (modified from Ricardi-Branco et al., 2009).

Hidrogeologia do Departamento de Geologia e Recursos Naturais, Instituto de Geociências, Universidade Estadual de Campinas. Estas amostras foram coletadas por Ricardi-Branco et al. (2009), com a finalidade de analisar a composição taxonômica das acumulações de macrorrestos vegetais que se encontram nas margens dos rios da bacia do Rio Itanhaém, especificamente nos rios Preto e Branco. A primeira amostra, apresenta 312 folhas/folíolos de angiospermas, e foi coletada no Rio Preto, em uma acumulação de macrorrestos vegetais que se depositou durante o ano de 1957 (Tabela 1; Figura 1). Em contrapartida, a segunda amostra, apresenta 619 folhas/
Tabela 1. Localização e período de deposição das acumulações de macrorrestos vegetais amostradas na Bacia do Rio Itanhaém (Ricardi-Branco et al., 2009).

Table 1. Location and deposition period of the plant macrodebris accumulations sampled in the Itanhaém River Basin (Ricardi-Branco et al., 2009).

\begin{tabular}{ccc}
\hline Localidades & Coordenadas geográficas & $\begin{array}{c}\text { Período de } \\
\text { deposição }\end{array}$ \\
\hline Rio Preto & $24^{\circ} 08^{\prime} 23,09^{\prime \prime}$ S 46 52'54,90”O & 1957 \\
Rio Branco & $24^{\circ} 05^{\prime} 04,00^{\prime \prime} \mathrm{S} 46^{\circ} 48^{\prime} 34,00^{\prime \prime} \mathrm{O}$ & $2004-2005$ \\
\hline
\end{tabular}


folíolos de angiospermas, e foi coletada no Rio Branco, em uma acumulação de macrorrestos vegetais que se depositou durante os anos 2004 e 2005 (Tabela 1; Figura 1).

\section{Reclassificação e identificação do material foliar}

O material foliar analisado foi inicialmente reclassificado em morfotipos, com base em sua arquitetura foliar, seguindo o manual de Ellis et al. (2009) e o esquema de classificação do DMNS (2005). Posteriormente, a partir de sua arquitetura foliar, foi novamente identificado, com o apoio de uma coleção de referência das espécies mais representativas da área de estudo (Ricardi-Branco et al., 2009), listas de vegetação (Lamberti, 1969; Garcia, 2003; IF, 2007) e herbários virtuais (http://fm1.fieldmuseum.org/ vrrc/, http://www.herbariovirtualreflora.jbrj.gov.br/, http:// www.tropicos.org/).

\section{Reconstrução da TMA e da PMA}

A reconstrução da TMA foi realizada com base na Análise da Margem Foliar. Este método relaciona a proporção de espécies de dicotiledôneas lenhosas sem dentes de uma flora com a TMA (Wilf, 1997; Kennedy et al., 2014). Devido a que esta correlação não é globalmente uniforme, já que o tipo de margem foliar num conjunto florístico está submetido a restrições filogenéticas e históricas (Kennedy et al., 2014), é recomendável a utilização de uma calibração no contexto de uma história fitogeográfica compartilhada (Hinojosa et al., 2011). Por esta razão, a equação selecionada (Tabela 2) foi uma desenvolvida a partir de um banco de dados que contém somente locais da zona tropical da América do Sul (Hinojosa et al., 2011).

Por sua vez, a reconstrução da PMA foi realizada com base na Análise da Área Foliar. Este método relaciona a área foliar média de uma flora com a PMA (Wilf et al., 1998). A diferença da Análise da Margem Foliar, a história evolutiva parece afetar menos o tamanho da folha que o tipo de margem foliar (Royer, 2012). Por esta razão, a equação selecionada (Tabela 2) não foi uma desenvolvida a partir de um banco de dados que contém somente locais da América do Sul, senão uma que foi desenvolvida a partir de um banco de dados que contém somente locais da zona tropical do mundo (Jacobs \& Herendeen, 2004).

O erro das reconstruções da TMA e PMA é conhecido ao comparar os valores obtidos com os valores reais para o momento de deposição das amostras de material foliar. Devido à inexistência de uma estação meteorológica na área de estudo, os dados de TMA e PMA, para o momento de deposição das amostras, foram tomados da literatura especializada. Como não foram encontrados dados específicos para os períodos de deposição, foram assumidos para o período de 1957 os valores de TMA e PMA do período de 1955-1964 (Lamberti, 1969), e para o período de 2004-2005 os valores de TMA e PMA atuais (IPT/PMI, 2012). Devido que os dados de TMA e PMA não são específicos, para os períodos de deposição, de antemão, já existe um fator de erro, ao momento de comparar os valores obtidos com os valores assumidos como reais. Este fator de erro pode ser mínimo, e pode estar dentro do erro das reconstruções.

\section{RESULTADOS}

\section{Reclassificação e identificação do material foliar}

A análise detalhada da arquitetura foliar do material presente na amostra que foi coletada no Rio Preto, permitiu distinguir 21 morfotipos, dos quais somente dez foram identificados taxonomicamente (Tabelas 3-4). Em contrapartida, a análise detalhada da arquitetura foliar do material presente na amostra que foi coletada no Rio Branco, permitiu distinguir 17 morfotipos, das quais somente sete foram identificados taxonomicamente (Tabelas 3-4).

\section{Reconstrução da TMA e da precipitação PMA}

A análise do tipo de margem e área foliar de 20 dos 21 morfotipos distinguidos na amostra coletada no Rio Preto, permitiu obter a proporção de espécies sem dentes, assim

Tabela 2. Equações baseadas na Análise da Margem Foliar e Análise da Área Foliar utilizadas para a reconstrução da temperatura média anual (TMA) e a precipitação média anual (PMA) para o momento de deposição das acumulações de macrorrestos vegetais amostradas na Bacia do Rio Itanhaém. Símbolos: a, número de locais; ' , coeficiente de determinação; ${ }^{\text {c }}$, erro padrão do modelo; ${ }^{\text {d }}$, região geográfica onde foram coletadas as amostras; ${ }^{\text {e, }}$ proporção de espécies sem dentes; ${ }^{\mathrm{f}}, \log$ natural da área foliar calculado de acordo com Wilf et al. (1998): $M \ln A=\Sigma a_{\mathrm{i}} p_{\mathrm{i}}\left(a_{\mathrm{i}}=\right.$ sete médias das áreas do log natural das classes de tamanho de Webb (1959), e $p_{\mathrm{i}}=$ a proporção de espécies em cada uma das classes de tamanho).

Table 2. Equations based on the Leaf Margin Analysis and Leaf Area Analysis used to the reconstruction of the mean annual temperature (TMA) and the mean annual precipitation (PMA) for the moment of deposition of the plant macrodebris accumulations sampled in the Itanhaém river basin. Symbols: a ${ }^{\text {, number }}$ of localities; ${ }^{\text {b }}$, coefficient of determination; ${ }^{\mathrm{c}}$, standard error of the model; ${ }^{\mathrm{d}}$, geographic region where the samples were collected; ${ }^{\mathrm{e}}$, proportion of untoothed species; ${ }^{\mathrm{f}}$, natural logarithm $\mathrm{f}$ the leaf area calculated according to Wilf et al. (1998): $M \ln A=\Sigma a_{\mathrm{i}} p_{\mathrm{i}}\left(a_{\mathrm{i}}=\right.$ seven means of the areas of the natural logarithm of the Webb (1959) size classes, and $p_{\mathrm{i}}=$ the proportion of species in each size class).

\begin{tabular}{lccccc}
\hline \multicolumn{1}{c}{ Equação } & $n^{\mathrm{a}}$ & $r^{2 \mathrm{~b}}$ & $E P^{\mathrm{c}}$ & Região $^{\mathrm{d}}$ & \\
\hline Análise da Margem Foliar & & & & & Fonte \\
\hline TMA $=23,42 E^{\mathrm{e}}+3,60$ & 44 & 0,48 & 3,5 & $\begin{array}{l}\text { Zona tropical da América do Sul (Bolívia, Brasil, } \\
\text { Colômbia, Equador, Guiana, Peru e Venezuela) }\end{array}$ & Hinojosa et al. (2011) \\
\hline Análise da Área Foliar & & & & & Jocobs \& Herendeen \\
\hline $\operatorname{lnPMA}=2,566+0,309 M \ln A^{\mathrm{f}}$ & 42 & 0,734 & - & Zonical da África e Bolívia & $(2004)$ \\
\hline
\end{tabular}


Tabela 3. Número de morfotipos e táxons identificados nas amostras de macrorrestos vegetais coletadas na Bacia do Rio Itanhaém. Símbolo: a, Ricardi-Branco et al. (2009).

Table 3. Number of morphotypes and taxa identified in the samples of plant macrodebris accumulations collected in the Itanhaém River Basin. Symbol: a, Ricardi-Branco et al. (2009).

\begin{tabular}{lcccc}
\hline & \multicolumn{2}{c}{$\begin{array}{c}\text { Rio Preto } \\
(1957)\end{array}$} & \multicolumn{2}{c}{$\begin{array}{c}\text { Rio Branco } \\
(2004-2005)\end{array}$} \\
\cline { 2 - 6 } & $\begin{array}{c}\text { Estudo } \\
\text { prévio }^{\text {a }}\end{array}$ & $\begin{array}{c}\text { Presente } \\
\text { estudo }\end{array}$ & $\begin{array}{c}\text { Estudo } \\
\text { prévio }^{\mathrm{a}}\end{array}$ & $\begin{array}{c}\text { Presente } \\
\text { estudo }\end{array}$ \\
\hline Total de morfotipos & 14 & 21 & 13 & 17 \\
Total de taxa identificados & 6 & 10 & 6 & 7 \\
\hline $\begin{array}{l}\text { Número total de folhas/ } \\
\text { folíolos de angiospermas }\end{array}$ & \multicolumn{2}{c}{312} & \multicolumn{2}{c}{619} \\
\hline
\end{tabular}

como a proporção em cada classe de tamanho e o logaritmo natural da área foliar (Tabelas 5-6). Estes valores permitiram reconstruir a TMA e a PMA para o momento de sua deposição (Tabela 5). Os valores obtidos subestimaram o valor real da TMA, em $1,6^{\circ} \mathrm{C}$, e da PMA, em $620 \mathrm{~mm}$ (Tabela 5). A análise do tipo de margem e área foliar de 16 dos 17 morfotipos distinguidos na amostra coletada no Rio Branco, permitiu obter a proporção de espécies sem dentes, assim como a proporção em cada classe de tamanho e o logaritmo natural da área foliar (Tabelas 5-6). Estes valores permitiram reconstruir a TMA e a PMA para o momento de sua deposição (Tabela 5). $\mathrm{O}$ valor de TMA obtido sobrestimou o valor real em $0,1^{\circ} \mathrm{C}$, e o valor de PMA obtido subestimou o valor real em $960 \mathrm{~mm}$ (Tabela 5).

Tabela 4. Táxons identificados nas amostras de macrorrestos vegetais coletadas na Bacia do Rio Itanhaém. Símbolo: a , Ricardi-Branco et al. (2009).

Table 4. Taxa identified in the samples of plant macrodebris accumulations collected in the Itanhaém River Basin. Symbol: a, Ricardi-Branco et al. (2009).

\begin{tabular}{|c|c|c|c|c|}
\hline Famílias & Gêneros e espécies & Hábito & $\begin{array}{c}\text { Rio Preto } \\
\text { (1957) }\end{array}$ & $\begin{array}{c}\text { Rio Branco } \\
\text { (2004-2005) }\end{array}$ \\
\hline Calophyllaceae & Calophyllum brasiliense Cambess. & Árvore & $x$ & \\
\hline Chrysobalanaceae & Parinari sp. & Árvore & $x$ & \\
\hline Euphorbiaceae & Alchornea triplinervia (Spreng.) Müll. Arg & Árvore & $x$ & \\
\hline \multirow[t]{3}{*}{ Fabaceae } & Inga vera Willd. & Árvore & $x$ & $x$ \\
\hline & Platymiscium sp. & Árvore & $x$ & \\
\hline & Senna sp. $^{\mathrm{a}}$ & Árvore & & $\times$ \\
\hline \multirow[t]{2}{*}{ Lauraceae } & Nectandra sp. ${ }^{a}$ & Árvore & & $x$ \\
\hline & Ocotea sp. ${ }^{a}$ & Árvore & $x$ & \\
\hline \multirow[t]{2}{*}{ Melastomataceae } & Miconia cinnamomifolia (DC.) Naudin & Árvore & & $x$ \\
\hline & Tibouchina sellowiana (Cham.) Cogn. & Árvore & & $x$ \\
\hline \multirow[t]{2}{*}{ Myrtaceae } & Myrcia sp. ${ }^{\mathrm{a}}$ & Árvore & $x$ & \\
\hline & Myrtaceae sp. & Árvore & & $x$ \\
\hline \multirow[t]{2}{*}{ Poaceae } & 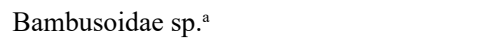 & Erva & $x$ & \\
\hline & Poaceae sp. ${ }^{a}$ & Erva & & $x$ \\
\hline Rubiaceae & Faramea sp. ${ }^{\mathrm{a}}$ & Árvore & $\times$ & \\
\hline Sapindaceae & Paullinia sp. ${ }^{\mathrm{a}}$ & Trepadeira & $x$ & \\
\hline
\end{tabular}

Tabela 5. Estimativa da temperatura média anual (TMA) e da precipitação média anual (PMA), para o momento de deposição das acumulações de macrorrestos vegetais amostradas na Bacia do Rio Itanhaém, com base na Análise da Margem Foliar e Análise da Área Foliar. Símbolos: a , proporção de espécies sem dentes; ${ }^{\text {b }}$, dados climáticos do município de Itanhaém para o período de 1955-1964 (Lamberti, 1969); ${ }^{\text {c }}$ log natural da área foliar calculado de acordo com Wilf et al. (1998); ' , dados climáticos atuais do município de Itanhaém (IPT/PMI, 2012).

Table 5. Estimate of the mean annual temperature (TMA) and the mean annual precipitation (PMA) for the moment of deposition of the plant macrodebris accumulations sampled in the Itanhaém river basin, based on the Leaf Margin Analysis and Leaf Area Analysis. Symbols: a, proportion of untoothed species; b, climatic data from municipality of Itanhaém for the period of 1955-1964 (Lamberti, 1969); ${ }^{\text {, }}$, natural logarithm of leaf area calculated according to Wilf et al. (1998); ' , current climate data from municipality of Itanhaém (IPT/PMI, 2012).

\begin{tabular}{|c|c|c|c|}
\hline $\begin{array}{l}\text { Rio Preto } \\
\text { (1957) }\end{array}$ & & $\begin{array}{l}\text { Rio Branco } \\
(2004-2005)\end{array}$ & \\
\hline Número de morfotipos & 20 & Número de morfotipos & 16 \\
\hline$E^{\mathrm{a}}$ & 0,850 & $E$ & 0,875 \\
\hline TMA $\left({ }^{\circ} \mathrm{C}\right)$ estimada (desvio da média $\left.1955-1964\right)^{\mathrm{b}}$ & $23,5(-1,6)$ & TMA $\left({ }^{\circ} \mathrm{C}\right)$ estimada (desvio da média atual) ${ }^{\mathrm{d}}$ & $24,1(+0,1)$ \\
\hline$M \ln A^{\mathrm{c}}$ & 6,90 & $M \ln A$ & 7,08 \\
\hline PMA (mm) estimada (desvio da média 1955-1964) ${ }^{\mathrm{b}}$ & $1097(-620)$ & PMA (mm) estimada (desvio da média atual) ${ }^{\mathrm{d}}$ & $1160(-960)$ \\
\hline
\end{tabular}


Tabela 6. Dados da área foliar das folhas/folíolos presentes nas amostras de macrorrestos vegetais coletadas na Bacia do Rio Itanhaém. Símbolos: a classes de tamanho segundo Webb (1959); a área foliar é calculada de acordo com Cain et al. (1956): $\mathrm{AF}=\mathrm{C} \times \mathbf{L} \times 0,67$ ( $\mathbf{A F}=$ área foliar, $\mathbf{C}=$ comprimento, $\mathbf{L}=$ largura); ${ }^{\text {b }}$, proporções relativas; ${ }^{\text {c }}$, média da área log natural para cada classe de tamanho; ${ }^{\mathrm{d}}, \log$ natural da área foliar calculado de acordo com Wilf et al. (1998), onde $M \ln A=\Sigma a_{i} p_{i}$.

Table 6. Leaf area data of the leaves/leaflets present in the samples of plant macrodebris accumulations collected in the Itanhaém River Basin. Symbols: a, size classes according to Webb (1959); the leaf area being calculated according to Cain et al. (1956): $\mathbf{L A}=\mathrm{L} \times \mathrm{W} \times 0,67(\mathbf{L A}=$ leaf area, $\mathbf{L}=1$ length, $\mathbf{W}=$ width); ${ }^{\mathbf{b}}$, relative ratios; ${ }^{\mathbf{c}}$, mean of the natural logarithm area for each size class; ${ }^{\mathrm{d}}$, natural logarithm of the leaf area calculated according to Wilf et al. (1998), where $M \ln A=\Sigma a_{i} p_{i}$.

\begin{tabular}{|c|c|c|c|c|c|c|}
\hline \multirow[b]{2}{*}{ Classe de tamanho ${ }^{\mathrm{a}}$} & \multirow{2}{*}{$\begin{array}{l}\text { Faixa de classe de } \\
\text { tamanho }\left(\mathrm{cm}^{2}\right)\end{array}$} & \multicolumn{2}{|c|}{$p_{\mathrm{i}}$ Classes de tamanho ${ }^{\mathrm{b}}$} & \multirow[b]{2}{*}{$a_{\mathrm{i}}^{\mathrm{c}}$} & \multicolumn{2}{|c|}{$M \ln A^{\mathrm{d}}\left(a_{i} p_{\mathrm{i}}\right)$} \\
\hline & & $\begin{array}{l}\text { Rio Preto } \\
\text { (1957) }\end{array}$ & $\begin{array}{l}\text { Rio Branco } \\
(2004-2005)\end{array}$ & & $\begin{array}{l}\text { Rio Preto } \\
\text { (1957) }\end{array}$ & $\begin{array}{l}\text { Rio Branco } \\
\text { (2004-2005) }\end{array}$ \\
\hline Leptofilo & $\leq 0,25$ & 0,00 & 0,00 & 2,12 & 0,00 & 0,00 \\
\hline Nanofilo & $>0,25-2,25$ & 0,04 & 0,07 & 4,32 & 0,18 & 0,32 \\
\hline Microfilo & $2,25-20,25$ & 0,67 & 0,52 & 6,51 & 4,34 & 3,38 \\
\hline Notofilo & $20,25-45,00$ & 0,25 & 0,30 & 8,01 & 2,00 & 2,37 \\
\hline Mesofilo & $45,00-182,25$ & 0,04 & 0,11 & 9,11 & 0,38 & 1,01 \\
\hline Macrofilo & $182,25-1640,20$ & 0,00 & 0,00 & 10,90 & 0,00 & 0,00 \\
\hline \multirow[t]{2}{*}{ Megafilo } & $>1640,20$ & 0,00 & 0,00 & 13,10 & 0,00 & 0,00 \\
\hline & & & & $\Sigma a_{i} p_{\mathrm{i}}$ & 6,90 & 7,08 \\
\hline
\end{tabular}

\section{DISCUSSÃO E CONCLUSÕES}

O número de morfotipos distinguidos em cada uma das amostras de macrorrestos vegetais analisadas é superior ao identificado previamente por Ricardi-Branco et al. (2009) (Tabela 3), mas inferior ao mínimo de 25-30 espécies recomendado por Wilf (1997), Wilf et al. (1998), Jacobs \& Herendeen, (2004) e Burnham et al. (2005) para realizar a reconstrução dos principais parâmetros climáticos, TMA e PMA, com base na fisionomia foliar. Apesar disto, levou-se a cabo a reconstrução da TMA e PMA, já que Ricardi-Branco et al. (2015) demonstraram que isto também é possível com um número inferior de espécies ao mínimo recomendado.

Geralmente, o material foliar das acumulações de macrorrestos vegetais que se encontram ao longo dos rios preserva as características da margem foliar, graças à rápida taxa de sedimentação e/ou baixa taxa de decomposição (Burnham et al., 2001; Ricardo-Branco et al., 2009). Por esta razão, o material foliar das acumulações de macrorrestos vegetais da Bacia do Rio Itanhaém permitiu a reconstrução da TMA da área para o momento de sua deposição (Tabela 5). As reconstruções da TMA subestimaram o valor real da TMA em $1,6^{\circ} \mathrm{C}$, e o sobrestimaram em $0,1^{\circ} \mathrm{C}$ (Tabela 5). Em geral, as reconstruções da TMA na região tropical a partir do material foliar presente nas acumulações de macrorrestos vegetais que se encontram ao longo dos rios tendem a subestimar o valor real em 2,5 a $5^{\circ} \mathrm{C}$ (Burnham et al., 2001), devido a maior proporção de espécies com margem com dentes que está presente neste ambiente (Burnham, 1989; Burnham et al., 2001; Greenwood, 2005). Além disso, as reconstruções da TMA estão condicionadas pela composição florística (Greenwood, 1992) e pelo habitat e hábito de procedência do material foliar (Burnham et al., 2001; Greenwood, 2005), assim como pelo viés introduzido pelos processos tafonômicos e de coleta das amostras (Greenwood, 1991, 1992, 2005; Burnham et al., 2005; Ricardi-Branco et al., 2015).

Por outro lado, temos que as reconstruções da PMA a partir do material foliar das acumulações de macrorrestos vegetais da Bacia do Rio Itanhaém subestimaram o valor real, especificamente em 620 e $960 \mathrm{~mm}$ (Tabela 5). O material foliar das acumulações de macrorrestos vegetais que se encontram ao longo dos rios também preserva as características da área foliar, mas geralmente estas não permitem a reconstrução da PMA com muita precisão (Ellis \& Johnson, 2013). As reconstruções da PMA, em geral, tendem a subestimar o valor real em mais de $400 \mathrm{~mm}$ (Burnham et al., 2001), devido à má representatividade das folhas com maior área foliar das árvores do dossel (Greenwood, 1991, 1992; Burnham, 1994), ao transporte que sofrem as folhas e ao ambiente deposicional (Ellis \& Johnson; 2013).

A ocorrência e a intensidade de El Niño, nas regiões tropicais e de latitudes médias, podem influenciar na dinâmica das precipitações (Marengo, 2007; Costa, 2012). Na região Sudeste de Brasil, não é observada uma influência clara de El Niño sobre as precipitações, mas sim no aumento da temperatura (Minuzzi et al., 2006; Marengo, 2007). Durante o período de deposição das acumulações de macrorrestos vegetais da Bacia do Rio Itanhaém analisadas, graças à influência de El Niño na área, foi gerado o material foliar necessário para a formação destas acumulações (RicardiBranco et al., 2020). Apesar disto, não houve nenhuma influência de El Niño sobre a TMA e a PMA (Marengo, 2007; Costas, 2012). Por esta razão, a reconstrução da TMA e da PMA, não é influenciada pela ocorrência e intensidade de El Niño.

Os erros obtidos no momento de reconstruir a TMA e a PMA, a partir do material foliar das acumulações de macrorrestos vegetais da Bacia do Rio Itanhaém, em conjunto 
aos erros obtidos em estudos prévios levados a cabo na região tropical, permitem reconstruir e interpretar melhor a TMA e a PMA a partir de associações fósseis de angiospermas, sobretudo daquelas associações de folhas fósseis, que apresentam semelhança com a vegetação que está presente na Bacia do Rio Itanhaém e que foram depositadas em sistema deposicional fluvial meandrante.

\section{AGRADECIMENTOS}

Os autores agradecem à Fundação de Amparo à Pesquisa do Estado de São Paulo (FAPESP; processos 2010/20379-6 e 2013/22729-2), pelo apoio financeiro, à Coordenação de Aperfeiçoamento de Pessoal de Nível Superior (CAPES) e ao Conselho Nacional de Desenvolvimento Científico e Tecnológico $(\mathrm{CNPq})$, pelas bolsas concedidas. Os autores também expressam seus agradecimentos a A. Pinilla, pela ajuda no trabalho de laboratório, e aos avaliadores anônimos e aos editores, A.M. Ribeiro e M.N. Ritter, pelas valiosas sugestões que ajudaram a melhorar o manuscrito.

\section{REFERÊNCIAS}

Amaral, P.G.C.; Ledru, M.P.; Ricardi-Branco, F. \& Giannini, P.C.F. 2006. Late Holocene development of a mangrove ecosystem in southeastern Brazil (Itanhaém, state of São Paulo). Palaeogeography, Palaeoclimatology, Palaeoecology, 241:608-820. doi:10.1016/j.paleo.2006.04.010

Branco, F.R.; Pereira, S.Y.; Branco, F.C. \& Pereira, P.R.B. 2011. Accumulation of bio debris and its relation with the underwater environment in the estuary of Itanhaém river, São Paulo state. In: I.A. Dar \& M.A. Dar (eds.) Earth and Environmental Sciences, InTech - Open Access, p. 565-590. doi:10.5772/26650

Burnham, R.J. 1989. Relationships between standing vegetation and leaf litter in a paratropical forest: Implications for paleobotany. Review of Palaeobotany and Palynology, 58:5-32. doi:10.1016/0034-6667(89)90054-7

Burnham, R.J. 1994. Patterns in tropical leaf litter and implications for angiosperm Paleobotany. Review of Palaeobotany and Palynology, 81:99-113. doi:10.1016/0034-6667(94)90129-5

Burnham, R.J. 1997. Stand characteristics and leaf litter composition of a dry forest hectare in Santa Rosa National Park, Costa Rica. Biotropica, 29:384-395. doi:10.1111/j.1744-7429.1997. tb00034.x

Burnham, R.J.; Ellis, B. \& Johnson, K.R. 2005. Modern tropical forest taphonomy: does high biodiversity affect paleoclimatic interpretations?. Palaios, 20:439-451. doi:10.2110/palo.2004. P04-60

Burnham, R.J.; Pitman, N.C.A.; Johnson, K.R. \& Wilf, P. 2001. Habitat-related error estimating temperatures from leaf margins in a humid tropical forest. American Journal of Botany, 88:10961102. doi: $10.2307 / 2657093$

Cain, S.A.; Castro, G.M.O.; Pires, J.M. \& Silva, N.T. 1956. Application of some phytosociological techniques to the Brazilian rain forest. American Journal of Botany, 43:911-941. doi:10.1002/j.1537-2197.1956.tb11184.x

Camargo, A.F.M.; Pereira, L.A. \& Pereira, A.M.M. 2002. Ecologia da bacia hidrográfica do rio Itanhaém. In: A. Schiaretti \& A.F.M. Camargo (eds.) Conceitos de bacias hidrográficas: teorias e aplicações, Editus, p. 239-256.
Costa, M.A. 2012. Avaliação dos eventos extremos de maré para os anos de 2002, 2003 e 2004, seus impactos e as alterações temporais (1987 - 2007) dos cenários da paisagem do município de Itanhaém - SP - Brasil. Programa de Pós-Graduação em Geociências, Universidade Estadual de Campinas, Tese de Doutorado, $149 \mathrm{p}$.

DMNS (Denver Museum of Nature \& Science). 2005. Guide to morphotyping fossil floras. Disponível em: http://www. paleobotanyproject.org/morphotyping.aspx; acesso em 20/01/2016.

Ellis, B.; Daly, D.C.; Hickey, L.J.; Johnson, K.R.; Mitchell, J.D.; Wilf, P. \& Wing, S.L. 2009. Manual of leaf architecture. Ithaca, Cornell University Press, 190 p.

Ellis, B. \& Johnson, K.R. 2013. Comparison of leaf samples from mapped tropical and temperate forests: implications for interpretations of the diversity of fossil assemblages. Palaios, 28:163-177. doi:10.2110/palo.2012.p12-073r

Garcia, R.J.F. 2003. Estudo florístico dos campos alto-montanos e matas nebulares do Parque Estadual da Serra do Mar, Núcleo Curucutu, São Paulo, SP, Brasil. Programa de Pós-graduação em Botânica, Universidade de São Paulo, Tese de Doutorado, 356 p.

Greenwood, D.R. 1991. The taphonomy of plant macrofossils. In: S.K. Donovan (ed.) The processes of fossilization, Belhaven Press, p. 145-169.

Greenwood, D.R. 1992. Taphonomic constraints on foliar physiognomic interpretations of Late Cretaceous and Tertiary Palaeoclimates. Review of Palaeobotany and Palynology, 71:149-190. doi:10.1016/0034-6667(92)90161-9

Greenwood, D.R. 2005. Leaf margin analysis: taphonomic constraints. Palaios, 20:499-506. doi:10.2110/palo.2004.P04-58

Hinojosa, L.F.; Pérez, F.; Gaxiola, A. \& Sandoval, I. 2011. Historical and phylogenetic constraints on the incidence of entire leaf margins: insights from a new South American model. Global Ecology and Biogeography, 20:380-390. doi:10.1111/j.14668238.2010.00595.x

IF (Instituto Florestal). 2007. Inventário florestal da vegetação natural do Estado de São Paulo: regiões administrativas de São José dos Campos (Litoral), Baixada Santista e Registro. São Paulo, SMA/Imprensa Oficial, 140 p.

IPT/PMI (Instituto de Pesquisas Tecnológicas do Estado de São Paulo/Prefeitura Municipal de Itanhaém). 2012. Atlas ambiental do município de Itanhaém. São Paulo, Imprensa Oficial, 92 p.

Jacobs, B.F. \& Herendeen, P.S. 2004. Eocene dry climated and woodland vegetation in tropical Africa reconstructed from fossil leaves from northern Tanzania. Palaeogeography, Palaeoclimatology, Palaeoecology, 213:115-123. doi:10.1016/j. palaeo.2004.07.007

Kennedy, E.M.; Arens, N.C.; Reichgelt, T.; Spicer, R.A.; Spicer, T.E.V.; Stranks, L. \& Yang, J. 2014. Deriving temperature estimates from Southern Hemisphere leaves. Palaeogeography, Palaeoclimatology, Palaeoecology, 412:80-90. doi:10.1016/j. palaeo.2014.07.015

Lamberti, A. 1969. Contribuição ao conhecimento da ecologia das plantas do manguezal de Itanhaém. São Paulo, Faculdade de Filosofia, Ciências e Letras, Universidade de São Paulo, 217 p. (Boletim 23). doi:10.11606/issn.2318-5988.v23i0p7-217

Marengo, J.A. 2007. Mudanças climáticas globais e seus efeitos sobre a biodiversidade: caracterização do clima atual $e$ definição das alterações climáticas para o território brasileiro ao longo do século XXI. Brasília, MMA, 212 p.

Minuzzi, R.B.; Sediyama, G.C.; Costa, J.M.N. \& Vianello, R.L. 2006. Influência do fenômeno climático El Niño no período chuvoso da região sudeste do Brasil. Geografia, 15:5-19. 
Ricardi-Branco, F.; Branco, F.C.; Garcia, R.F.; Faria, R.S.; Pereira, S.Y.; Portugal, R.; Pessenda, L.C. \& Pereira, P.R.B. 2009. Plant accumulations along the Itanhaém River Basin, southern coast of São Paulo state, Brazil. Palaios, 24:416-424. doi:10.2110/ palo.2008.p08-079r

Ricardi-Branco, F.; Ianniruberto, M.; Silva, A.M. \& Branco, F. 2011. Plant debris accumulations in the Preto river subbasin, Itanhaém, São Paulo, Brazil: insights from geotechnology. Palaios, 26:264-274. doi:10.2110/palo.2010.p10-125r

Ricardi-Branco, F.; Pereira, S.Y.; Souza, M.M.; Santiago, F.; Pereira, P.B.P.; Branco, F.C.; Ribeiro, V. \& Molina, K. 2015. Relationships among subaquatic environment and Leaf/ Palinomorph assemblages of the Quaternary Mogi-Guaçú River alluvial plain, SP, Brazil. In: M. Ramkumar; K. Kumaraswamy \& R. Mohanraj (eds.) Environmental management of river basin ecosystems, Springer International Publishing, p. 667-705. doi:10.1007/978-3-319-13425-3_30

Ricardi-Branco, F.; Rios, F.S. \& Pereira, S.Y. 2020. Actualistic taphonomy of plant remains in tropical forests of Southeastern Brazil. In: S. Martínez; A. Rojas \& F. Cabrera (eds.) Actualistic Taphonomy in South America, Cham, Springer, p. 111-138 (Topics in Geobiology 48). doi:10.1007/978-3-030-20625-3_7

Royer, D.L. 2012. Climate reconstruction from leaf size and shape: new developments and challenges. In: L.C. Ivany \& B.T. Huber (eds.) Reconstructing earth's deep-time climate-the state of the art in 2012, Bethesda, The Paleontological Society, p. 195-212 (Papers 18).

Spavorek, G.; Van Lier, Q.D.J. \& Neto, D.D. 2007. Computer assisted Koeppen climate classification: a case study for Brazil. International Journal of Climatology, 27:257-266. doi:10.1002/ joc. 1384

Veloso, H.P.; Rangel Filho, A.L.R. \& Lima, J.C.A. 1991. Classificação da vegetação brasileira adaptada a um sistema universal. Rio de Janeiro, Instituto Brasileiro de Geografia e Estatística, Departamento de Recursos Naturais e Estudos Ambientais, $124 \mathrm{p}$.

Webb, L.J. 1959. A physiognomic classification of Australian rain forest. Journal of Ecology, 47:551-570. doi:10.2307/2257290

Wilf, P. 1997. When are leaves good thermometers? A new case for leaf margin analysis. Paleobiology, 23:373-390. doi:10.1017/ S0094837300019746

Wilf, P.; Wing, S.L.; Greenwood, D.R. \& Greenwood, C.L. 1998. Using fossil leaves as paleoprecipitation indicators: an Eocene example. Geology, 26:203-206. doi:10.1130/00917613(1998)026<0203:UFLAPI>2.3.CO;2

Received in 17 December, 2019; accepted in 07 October, 2020. 